\title{
Effects of L-Dopa and Dopamine D2 Agonists on Recollection and Familiarity in Idiopathic Nondementing Parkinson's Disease
}

\author{
Nicola M. J. Edelstyn ${ }^{1 *}$, Thomas A. Shepherd ${ }^{1}$, Andrew R. Mayes ${ }^{2}$, Susan M. Sherman ${ }^{1}$, \\ Simon J. Ellis ${ }^{3,4}$ \\ ${ }^{1}$ School of Psychology, Keele University, Staffordshire, UK; ${ }^{2}$ School of Psychological Sciences, The University of Manchester, \\ Manchester, UK; ${ }^{3}$ University Hospital of North Staffordshire, Stoke-on-Trent, UK; ${ }^{4}$ Keele University, Staffordshire, UK. \\ E-mail: *n.edelstyn@psy.keele.ac.uk
}

Received October $8^{\text {th }}, 2011$; revised November $18^{\text {th }}, 2011$; accepted November $28^{\text {th }}, 2011$.

\begin{abstract}
Idiopathic nondementing Parkinson's disease (PD) is marked by progressive loss of dopaminergic neurons in the substantia nigra pars compacta and ventral tegmental area. Recent brain imaging work implicates these structures in dopamine modulated networks subserving episodic memory. These findings are of relevance to PD because they suggest that dopamine depletion contributes to the disease-dependent decline in episodic memory, and therefore, this decline should, at least partially, be remediated by dopaminergic medication. Recognition memory (RM), recollection and familiarity during recognition was examined in 17 PD patients, 12 of whom were medicated with a D2 dopamine agonist (pramipexole or ropinirole) and l-dopa, with a further 5 PD control patients on l-dopa but no D2 agonist. Memory was tested "ON" and, following a period of medication withdrawal, "OFF" and compared to a group of 14 matched healthy volunteers $(H V)$. The HVs were also tested twice in the absence of medication. The patients on the agonists PD showed significant impairments in recollection ON- and OFF-medication, whereas the l-dopa control patients exhibited a decline in OFF-recollection only. RM and familiarity were spared in both groups ON- and OFF-medication. These findings suggest that D2 dopamine agonists (combined with l-dopa) contribute to disease-dependent episodic memory impairment.
\end{abstract}

Keywords: Parkinson’s Disease, D2 Dopamine Agonists, L-Dopa, Recognition, Recollection, Familiarity

\section{Introduction}

We report an investigation of l-dopa and second generation nonergoline dopamine agonists ("D2 agonists") on the recollection of episodic details and the assessment of familiarity during recognition in patients with nondementing idiopathic Parkinson's disease (PD). This study builds on previous a previous report [1] of a selective impairment in recollection in moderate PD when optimally medicated with dopaminergic medication. Furthermore, the data indicated that the severity of the recollection deficit decreased when the same patients were tested in following a 12 - 14 hour over-night period of medication withdrawal. In contrast, familiarity showed relative sparing in both unmedicated and medicated conditions.

A dissociation between recollection and familiarity in medicated PD has been reported previously, although consensus is lacking regarding the direction of this dissociation. There are a number of reports [2-5] of a selective deficit in recollection; whereas others [6,7] have found a more pronounced impairment in familiarity. Recent evidence of a double dissociation between recollection and familiarity in the same study [8], albeit in different patient groups, implies that methodological differences between studies may contribute to the direction of the dissociation.

The suggestion that recollection and familiarity doubly dissociate in Parkinson's is consistent with the dual process view of recognition memory [9] and Aggleton and Brown's [10,11] still controversial neuroanatomical model of recognition memory. In this model, recollection is reliant on an extended hippocampal circuit whereas familiarity is dependent on a separate network, extending 
from the perirhinal cortex in the medial temporal lobe to the mediodorsal thalamus and frontal lobes.

The medial temporal lobe structures identified in Aggleton and Brown's model, and particularly the hippocampus, are dependent on dopaminergic modulation, and are therefore, also vulnerable to dopamine depletion, the hallmark pathology of PD.

Rodent studies show dopamine induces long-term potentiation (a form of synaptic plasticity believed to underlie memory storage) in both the CA1-3 fields of the hippocampus [12-15] and the perirhinal cortex [16-18], with D2 dopamine receptors critically involved in this process [19-21].

Brain imaging evidence indicates dopamine modulates activity in the substantia nigra pars compacta, and mesolimbic circuit (involving the ventral tegmental area and the hippocampus) during the acquisition of novel associative information [22-29] and signals about past and future rewards and punishments [30-32].

Episodic memory can also be manipulated by drugs which either block or promote dopamine activity. So, for example, injecting the dopamine D1 and D2 antagonist D-AP-5 into rodent hippocampus impairs maze learning [32-34]; whereas administration of D1 agonists, such as piribedil and bromocriptine, and SKF 38393 and SKF 81297, promotes spatial memory in young adult rats $[35,36]$ and aged rats [37]. In humans, l-dopa (100 mg plus $25 \mathrm{mg}$ carbidopa) has also been shown to increase verbal encoding in healthy young and older adults adults, as well as stroke patients [38-40].

The suggestion that routine dopaminergic medication used to control motor symptoms in PD has the capacity to affect cognitive function is well established. PD patients medicated with l-dopa show improved working memory [41-43], dual-tasking [44,45], task-switching [46-48], feedback-based sequence learning [49], and verbal fluency [50] compared to their unmedicated performance. However, l-dopa can also impair self-ordered pointing and conditional associative learning [50], probabilistic reversal learning [46], extinction learning [51], betting strategies in a gambling task [47] and the Wisconsin Card Sorting Test [52].

It's been proposed that the direction in which l-dopa affects a particular cognition is dependent on the integrity of the underlying dopamine-modulated neural circuitry. According to the l-dopa overdose hypothesis, the requisite dopaminergic state necessary to control motor symptoms has the potential to move the same patient towards and away from their optimum for certain cognitive functions [46,50,53,54]. The relationship between the efficiency of neuronal activity and the state of dopaminergic modulation is represented by an inverted
U-shaped curve with cognitive functions declining with deviation away from optimum dopamine levels, indicated by the centre of the curve. Accordingly, where de novo patients show impairments in working memory for example but medicated patients do not [55], l-dopa serves to restore depleted dopamine levels in the neural structures subserving working memory. By contrast, when l-dopa is withdrawn and formally deficient cognitions, such as probabilistic reversal learning, are remediated, then the effect of l-dopa is to "overdose" neural circuits where dopamine modulation remains relatively spared.

D2 agonists are frequently combined with l-dopa in PD. Their effects have been less extensively studied compared to l-dopa, although they have been linked to a "dopamine dysregulation syndrome", where some patients engage in risk-taking behaviour such as pathological gambling and hypersexuality [56,57]. There is also some evidence that D2 agonists may supportworking memory and task switching, but impairs probabilistic reversal learning [46,58].

In sum, the findings from animal research, pharmacological and brain imaging studies of healthy volunteers support a role for dopamine modulation of hippocampal, and to a lesser extent perirhinal, memory processes. Based on the underlying level of dopamine activity in these memory structures, as well as prefrontal areas which support strategic memory, the l-dopa overdose hypothesis predicts differential effects on memory. Studies of dopaminergic medication and executive function in PD indicate that both l-dopa and D2 agonists have the potential to both remediate and impair executive function. Although only one study to date has examined the effect of dopaminergic medication on episodic memory, there is good reason, based on the evidence reviewed, to suspect that the recollection of episodic details may also be affected by dopaminergic medication.

The aim of our investigation was to investigate the effect of D2 agonists on the recollection of episodic details and the assessment of familiarity during recognition. Because D2 agonists are frequently combined with l-dopa, a separate control group of patients on l-dopa but not agonists will also be assessed.

The procedure adopted in this study is the controlled medication withdrawal procedure, with memory assessed in the same patients when unmedicated or in an OFF and again after patients have taken their routine medication (termed medicated or ON state). This procedure is less prone to the confounds of differences in disease severity compared to the alternative procedure of comparing de novo, i.e. never medicated, patients with the same individuals at a later stage after dopaminergic medication administration, or a different already-treated group. 
The first set of predictions applies to the OFF-state, where we expect patients in both the D2 agonist group and the l-dopa group (patients on l-dopa monotherapy or in combination with an enzyme inhibitor) to exhibit a dissociation between deficient recollection and preserved familiarity.

The second set of predictions applies to the ONmedication state. The l-dopa group is expected to show enhanced recollection. According to the l-dopa overdose hypothesis, patients in the l-dopa group should show an enhancement of recollection. Because the mode of action of D2 agonists on cognition is less well formulated, the following predictions are driven by empirical observations rather than theory. Inspection of medication regimen of patients reported in our previous study [1], indicated that l-dopa plus a D2 agonist failed to remediate recollection and if anything, served to increase the magnitude of the OFF-medication recollection impairment further. We also expect familiarity to be spared in line with previous empirical results $[1,2,4]$ and the proposal that this type of memory is reliant on a different nondopamine-dependent neural pathway to that which supports recollection.

Finally, within-group comparisons of recollection memory should reveal significant differences ON and OFF medication, with patients in the D2 agonist group displaying significantly improved OFF-compared to ONrecollection rates. By contrast, the direction of this effect should be reverse in the l-dopa group, with improved recollection ON compared to OFF-medication.

\section{Participants and Methods}

\subsection{Participants}

A group of seventeen patients in the mild to moderate stages of Parkinson's (mean medicated Hoehn and Yahr [59] [HY] disease severity rating 2.6, range: 1 - 4) were recruited from the Parkinson's disease outpatient clinic in the Department of Neurology, University Hospital of North Staffordshire. During a clinical interview with a consultant neurologist (SJE), patients were screened for adverse clinical events or issues that might affect performance (e.g. deep brain stimulation, dyskinesias, dopamine dysregulation syndrome, drastic medication changes, fatigue, distress, commenced dopaminergic medication 2 months prior to commencement of the study).

Twelve patients of this initial cohort were taking a D2 agonist, either pramipexole or ropinirole, combined with l-dopa. The remaining five patients were taking l-dopa with/without an enzyme inhibitor only. Both patient subgroups were matched for age $(t=-1.57, p=0.14)$, pre- morbid IQ (National Adult Reading Test [60], [NART]: $t$ $=-0.20, p=0.84$ ), current levels of functioning (MiniMental State Examination [61], [MMSE]: $t=-0.09, p=$ 0.93; The Cambridge Examination for Mental Disorders of the Elderly [62], [CAMCOG]: $t=1.23, p=0.24$ ), depression rating (Hamilton Depression Inventory [63], [HDI]: $t=0.25, p=0.81$ ); motor symptom severity OFFand ON-medication (Unified Parkinson's Disease Rating Scale [64], [UPDRS]: $t=0.73, p=0.48$ and $t=0.17, p=$ 0.87 , respectively), l-dopa equivalent dose $(t=-1.73, p=$ $1.00)$, disease duration $(t=-0.11, p=0.91)$ and HY score $(t=0.27, p=0.79)$. The raw data are presented in Table 1.

A single group of 14 healthy volunteers (HV) served as controls for the D2 agonist and l-dopa patient subgroups. The HV matched both patient subgroups for age (D2 agonist: $t=0.02, p=0.98$; l-dopa: $t=-1.43, p=1.0$ ), premorbid IQ (D2 agonist: $t=-0.32, p=0.75$; l-dopa: $t=$ $-0.25, p=0.8$ ), current levels of functioning on the MMSE and CAMCOG (D2 agonist: $t=-0.93, p=0.36$ and $t=1.16, p=0.25$, respectively; l-dopa: $t=-0.65, p=$ 0.52 and $t=2.0, p=0.054$, respectively) but not depression (D2 agonist: $t=-2.23, p=0.03$; l-dopa: $t=-2.91, p$ $=0.01$ ). The raw data for the HV groups are also presented in Table 1.

Exclusion criteria for all participants included a MMSE score of 25 or less, presence of neurological or psychiatric history (apart from PD in the index groups), history of substance abuse (including alcoholism), antidepressants, learning difficulty (including dyslexia), or English as a second language.

\subsection{Procedure}

Two versions of a "yes/no" recognition memory test ("RMT1" and "RMT2") were constructed from a pool of three hundred and twenty 4-6 letter words, and matched for word frequency $($ RMT1: mean $=115.4$ per million, range 1 - 1461, SD = 160.51; RMT2: mean = 115.65 per million, range $1-1789, \mathrm{SD}=160.87$ ) concreteness $($ RMT1: mean $=467.9$, SD = 6.36; RMT2: mean $=466.9$, $\mathrm{SD}=8.79$ ) and imageability (RMT1: mean $=487.2$, SD = 6.08; RMT2: mean = 486.5, SD = 5.66), using published norms $[65,66]$, and for relative contribution of familiarity and recollection at test.

At study, participants saw a mixture of 80 high and low frequency words for 3-seconds each (3-second interstimulus interval) and made a judgement as to whether the word was pleasant, unpleasant or neutral. Immediately after completing the study phase, recognition using the yes/no procedure was tested by presenting each of the studied words (targets) randomly intermixed with 80 high and low frequency new words or lures, that were 
Table 1. Participant demographic, neuropsychological and clinical (patients only) features.

\begin{tabular}{|c|c|c|c|c|c|c|c|c|c|c|c|c|}
\hline & \multirow{2}{*}{ HDI } & \multirow{2}{*}{ Age } & \multirow{2}{*}{ MMSE } & \multirow{2}{*}{ NART } & \multirow{2}{*}{ CAMCOG } & \multirow{2}{*}{ HY } & \multirow{2}{*}{$\begin{array}{c}\text { Illness } \\
\text { Duration }\end{array}$} & \multicolumn{2}{|c|}{ UPDRS } & \multicolumn{3}{|c|}{ Medication } \\
\hline & & & & & & & & OFF & ON & l-dopa & D2 agonist ${ }^{\mathrm{a}, \mathrm{b}}$ & Inhibitor $^{c, d}$ \\
\hline \multicolumn{13}{|c|}{ Healthy Volunteers (HV) } \\
\hline HV 1 & 3 & 59 & 29 & 119 & 102 & & & & & & & \\
\hline HV 2 & 2.7 & 60 & 30 & 121 & 102 & & & & & & & \\
\hline HV 3 & 2.2 & 66 & 30 & 119 & 102 & & & & & & & \\
\hline HV 4 & 3 & 64 & 29 & 109 & 101 & & & & & & & \\
\hline HV 5 & 0.7 & 72 & 30 & 121 & 99 & & & & & & & \\
\hline HV 6 & 4 & 79 & 29 & 118 & 103 & & & & & & & \\
\hline HV 7 & 11 & 64 & 30 & 124 & 102 & & & & & & & \\
\hline HV 8 & 4.2 & 67 & 30 & 118 & 102 & & & & & & & \\
\hline HV 9 & 7 & 71 & 30 & 119 & 103 & & & & & & & \\
\hline HV 10 & 3 & 77 & 30 & 124 & 104 & & & & & & & \\
\hline HV 11 & 2 & 60 & 30 & 122 & 104 & & & & & & & \\
\hline HV 12 & 1 & 68 & 30 & 110 & 98 & & & & & & & \\
\hline HV 13 & 4.4 & 54 & 29 & 107 & 99 & & & & & & & \\
\hline HV 14 & 7 & 69 & 28 & 102 & 100 & & & & & & & \\
\hline MEAN & 4 & 66 & 29.57 & 116.6 & 101.5 & & & & & & & \\
\hline SD & 2.8 & 7 & 0.646 & 6.823 & 1.870829 & & & & & & & \\
\hline \multicolumn{13}{|c|}{$D 2$ agonist subgroup $(D A)$} \\
\hline DA 1 & 3 & 64 & 29 & 109 & 99 & 3 & 6 & 17 & 15 & 250 & $16^{\mathrm{a}}$ & $1^{\mathrm{c}}$ \\
\hline DA 2 & 4 & 71 & 30 & 127 & 98 & 2 & 6 & 10 & 10 & 200 & $7^{\mathrm{a}}$ & $1^{\mathrm{c}}$ \\
\hline DA 3 & 28 & 61 & 30 & 115 & 102 & 3 & 8.5 & 22 & 14 & 400 & $1^{\mathrm{b}}$ & 0 \\
\hline DA 4 & 9.8 & 77 & 29 & 117 & 98 & 3 & 7 & 15 & 16 & 500 & $16^{\mathrm{b}}$ & 0 \\
\hline DA 5 & 18 & 64 & 30 & 98 & 101 & 4 & 10 & 23 & 20 & 375 & $16^{\mathrm{b}}$ & 0 \\
\hline DA 6 & 14 & 65 & 28 & 113 & 103 & 2 & 5 & 8 & 6 & 300 & $24^{\mathrm{b}}$ & $200^{\mathrm{d}}$ \\
\hline DA 7 & 1 & 75 & 30 & 124 & 104 & 2.5 & 3 & 13 & 5 & 250 & $16^{\mathrm{b}}$ & 0 \\
\hline DA 8 & 3.8 & 62 & 29 & 126 & 103 & 2.5 & 4 & 7 & 4 & 250 & $9^{b}$ & 0 \\
\hline DA 9 & 4 & 59 & 30 & 126 & 100 & 2.5 & 2 & 11 & 11 & 100 & $11^{\mathrm{b}}$ & 0 \\
\hline DA 10 & 11 & 69 & 30 & 123 & 100 & 3 & 14 & 17 & 14 & 525 & $11^{\mathrm{b}}$ & 0 \\
\hline DA 11 & 6 & 68 & 30 & 114 & 100 & 2 & 3 & 9 & 6 & 100 & $12^{\mathrm{b}}$ & 0 \\
\hline DA 12 & 4 & 55 & 30 & 118 & 100 & 3 & 5 & 7 & 7 & 450 & $12^{\mathrm{b}}$ & 0 \\
\hline MEAN & 8.9 & 66 & 29.58 & 117.5 & 100.6667 & 2.71 & 5.63 & 13.3 & 10.7 & 308.3 & 12.8 & 0 \\
\hline SD & 7.8 & 7 & 0.63 & 7.93 & 2.06 & 0.58 & 3.24 & 5.58 & 5.14 & 143.2 & 5.94 & 0 \\
\hline \multicolumn{13}{|c|}{ L-dopa subgroup } \\
\hline L-dopa 1 & 7.5 & 65 & 30 & 125 & 98 & 1 & 3 & 9 & 8 & 300 & & \\
\hline L-dopa 2 & 8.5 & 63 & 29 & 109 & 99 & 2 & 4 & 6 & 6 & 250 & & \\
\hline L-dopa 3 & 6.5 & 73 & 30 & 124 & 102 & 2.5 & 6 & 1 & 1 & 400 & & \\
\hline L-dopa 4 & 8.4 & 79 & 29 & 107 & 98 & 3 & 8 & 17 & 16 & 412.5 & & \\
\hline L-dopa 5 & 7 & 76 & 30 & 123 & 100 & 4 & 12 & 17 & 17 & 1000 & & \\
\hline MEAN & 7.6 & 71 & 29.6 & 117.6 & 99.4 & 2.5 & 6.6 & 10 & 9.6 & 472.5 & & \\
\hline SD & 0.9 & 6.9 & 0.55 & 8.82 & 1.67 & 1.12 & 3.58 & 7 & 6.8 & 302.6 & & \\
\hline
\end{tabular}

Notes: Significantly different from ${ }^{1}$ healthy control group at ${ }^{*} p<0.05$; Abbreviations: $\mathrm{SD}=1$ standard deviation; MMSE $=$ Mini-Mental state examination; NART = National Adult Reading Test; HDI = Hamilton Depression Inventory; CAMCOG = The Cambridge Examination for Mental Disorders of the Elderly-Revised; HY = Hoehn and Yahr; UPDRS = Unified Parkinson's Disease Rating Scale; ON = medicated state; OFF = unmedicated state; dopamine D2 agonists: ${ }^{a}$ pramipexole, ${ }^{b}$ ropinirole. Enzyme inhibitor: ${ }^{\mathrm{M}} \mathrm{MOAI},{ }^{\mathrm{d}} \mathrm{COMT}$. 
matched to the targets for mean word frequency, concreteness and imageability. Each word was presented individually, and recognition judgements were made within a 3-second response window.

Correct identification of a target item was defined as a hit, whilst false recognition of a lure was termed a false alarm. Following each endorsement, irrespective of whether it was a hit or false alarm, participants made a subjective judgement of their recognition experience in terms of either feelings of familiarity without any recollection ("know" response) or a specific recollection of the item having been previously presented ("remember" response). The second stage was not time constrained. Participants were given practice trials prior to completing both RMT1 and RMT2, and regular checks were made throughout the test phase to ensure that participants maintained a full understanding of the criteria for making a remember/know decision.

To examine the effect of medication, patients were tested ON and OFF medication.

In the ON-condition, patients were tested in the morning, 2 hours after taking their first medication of the day. To produce the OFF-state, patients were assessed at the same time of day having delayed their first morning medication. The time since last medication was 12 - 14 hours. The HVs were also tested for 2 sessions, labelled "Blue" and "Green". This label emphasized that there was no difference in "treatment" between the two sessions: the "Blue" was yoked to the OFF session of the patients, the "Green" to the ON session. The order of RMT1 and RMT2 were counterbalanced across the ON/Green and OFF/Blue sessions, and the order of ON/Green and OFF/Blue sessions were counterbalanced across participants.

The study was approved by South Staffordshire NHS Research Ethics Committee.

\subsection{Performance Measures}

A correction has been made to the raw data to eliminate extreme scores [67]. It is assumed that recollection and familiarity are stochastically independent at retrieval, and therefore the independence formula has been applied to the corrected know data (Familiarity $=$ know/[1-remember]) [68]. Estimates of recognition memory and familiarity were then calculated using d', and recollection is reported as a threshold measure (hits rate minus false alarms).

\subsection{Analyses}

The estimates of RM, familiarity and recollection were analyzed using a series of 2 by 3 Analysis of Variance (ANOVA) with Group as the betweens subject factor
(HV versus the D2 agonist PD subgroup versus l-dopa control PD subgroup) and Condition the within subjects factor (OFF-medication/Blue versus ON-medication/ Green).

The distributions of depression scores of the HV and PD subgroups were markedly different and the variances of the groups were heterogeneous. However Analysis of Covariance with depression as a covariate weren't run because the mean depression score which would have been applied is above the score of any of the members of the comparison group.

\section{Results}

Raw hit and false alarm rates for recognition memory (RM), know and remember judgements in the OFFmedication/Blue and ON-medication/Green conditions by group are presented in Table 2 .

Estimates of RM, familiarity and recollection in the OFF-medication/Blue and ON-medication/Green conditions by group are presented in Table 3 and Figure 1 .

The first 2 by 3 ANOVA of RM (d') showed no main effects of either Group (HV versus PD, medication subgroups collapsed, $F[1,34]=2.14, p=0.15$ ) or Condition (OFF-medication/Blue and ON-medication/Green, $F[1,34]$ $=0.15, p=0.7)$, and the Interaction between Group and Condition was also not significant $(F[1,34]=0.01, p=$ 0.81 ). The second 2 by 3 ANOVA of familiarity (d') revealed the same pattern of results with no main effects of either Group (HV versus PD, medication subgroups collapsed, $F[1,34]=0.82, p=0.37)$ or Condition $(F[1,34]$ $=0.3, p=0.59)$, and the Interaction was again not significant $(F[1,34]=0.6, p=0.81)$. Taken together, these results do not give any indication that neither disease nor medication effect RM or familiarity in this cohort of PD patients.

The next 2 by 3 ANOVA which analysed recollection estimates revealed a main effect of Group (HV versus $\mathrm{PD}$, medication subgroups collapsed, $F[1,34]=6.99, p=$ 0.012 ), but neither Condition (OFF-medication/Blue and ON-medication/Green, $F F[1,34]=0.28, p=0.6$ ) nor the Interaction $(F[1,34]=0.17, p=0.69)$ were significant. Separate one-way ANOVA of OFF/Blue and ON/Green recollection rates with Group as the between-subjects variable showed a significant difference in OFF/Blue recollection $(F[2,35]=3.86, p=0.03)$ and ON/Green recollection approached significance $(F[2,35]=2.77, p=$ 0.08 ). A series of planned pair-wise comparisons revealed OFF-medication recollection to be significantly lower in the agonist patients $(p=0.035)$ and the l-dopa ( $p$ $=0.027$ ) compared to the HVs, there was no difference between the PD subgroups ( $p>0.05$ ). These findings indicate a general disease-dependent decline in recollection 
Table 2. Mean hit and false alarm rates for recognition memory, know and remember rates in ON/Green and OFF/Blue conditions by group.

\begin{tabular}{|c|c|c|c|c|c|c|c|c|c|c|c|c|}
\hline \multirow{3}{*}{ GROUP } & \multicolumn{4}{|c|}{ Recognition memory } & \multicolumn{4}{|c|}{ Know } & \multicolumn{4}{|c|}{ Remember } \\
\hline & \multicolumn{2}{|c|}{ OFF/Blue } & \multicolumn{2}{|c|}{ ON/Green } & \multicolumn{2}{|c|}{ OFF/Blue } & \multicolumn{2}{|c|}{ ON/Green } & \multicolumn{2}{|c|}{ OFF/Blue } & \multicolumn{2}{|c|}{ ON/Green } \\
\hline & HR & FAR & HR & FAR & HR & FAR & HR & FAR & HR & FAR & HR & FAR \\
\hline \multicolumn{13}{|c|}{ Healthy volunteer group $(n=14)$} \\
\hline Mean & 70.5 & 6.6 & 70.5 & 6.43 & 13.1 & 5.1 & 13.7 & 4.7 & 57.2 & 1.4 & 56.9 & 1.9 \\
\hline SD & 6.2 & 5.3 & 5.6 & 6.5 & 6.0 & 4.5 & 4.6 & 4.8 & 8.3 & 1.3 & 8.5 & 2.0 \\
\hline \multicolumn{13}{|c|}{ D2 agonist subgroup $(n=12)$} \\
\hline Mean & 59.4 & 5.1 & 60.3 & 6.1 & 15.3 & 3.9 & 19.0 & 4.8 & 45.3 & 1.3 & 42.4 & 1.6 \\
\hline SD & 12.2 & 4.2 & 13.4 & 4.6 & 5.7 & 4.3 & 9.1 & 3.3 & 16.4 & 1.7 & 18.2 & 2.3 \\
\hline \multicolumn{13}{|c|}{ I-dopa subgroup $(n=5)$} \\
\hline Mean & 58.80 & 6.20 & 64.20 & 8.8 & 19.20 & 4.40 & 20.60 & 5.80 & 38.60 & 1.80 & 43.60 & 2.80 \\
\hline SD & 12.28 & 7.56 & 8.59 & 10.45 & 4.09 & 4.22 & 15.37 & 8.26 & 16.14 & 3.49 & 17.36 & 5.22 \\
\hline
\end{tabular}

Notes and Abbreviations: $\mathrm{HR}=$ hit rate; $\mathrm{FAR}=$ false alarm rate; $\mathrm{SD}=1$ standard deviation; $\mathrm{OFF}=$ unmedicated state; $\mathrm{ON}=$ medicated state.

Table 3. Estimates of ON-medication/Green and OFF-medication/Blue recognition, familiarity and recollection by participant group.

\begin{tabular}{|c|c|c|c|c|c|c|}
\hline & \multicolumn{2}{|c|}{ RM (d') } & \multicolumn{2}{|c|}{ Familiarity (d') } & \multicolumn{2}{|c|}{ Recollection (HR-FAR) } \\
\hline & OFF/Blue & ON/Green & OFF/Blue & ON/Green & OFF/Blue & ON/Green \\
\hline \multicolumn{7}{|c|}{ Healthy volunteer group $(n=14)$} \\
\hline MEAN & 2.22 & 2.28 & 1.61 & 1.70 & 0.66 & 0.65 \\
\hline SD & 0.76 & 0.96 & 0.71 & 0.80 & 0.15 & 0.15 \\
\hline \multicolumn{7}{|c|}{ D2 agonist subgroup $(n=12)$} \\
\hline MEAN & 2.24 & 2.22 & 1.63 & 1.72 & $0.52 *$ & $0.50 *$ \\
\hline SD & 0.70 & 0.78 & 0.53 & 0.57 & 0.20 & 0.22 \\
\hline \multicolumn{7}{|c|}{$L$-dopa subgroup $(n=5)$} \\
\hline MEAN & 2.26 & 2.47 & 1.68 & 1.86 & $0.45^{*}$ & 0.50 \\
\hline SD & 0.78 & 0.87 & 0.73 & 0.42 & 0.19 & 0.21 \\
\hline
\end{tabular}

Notes: significance level: ${ }^{\mathrm{p}}$ < 0.05; Abbreviations: $\mathrm{SD}=1$ standard deviation; $\mathrm{ON}=$ medicated state; OFF = unmedicated state.

independent of medication subgroup. ON-recollection was also significantly lower in the agonist patients compared to the HVs $(p=0.036)$, but not in the l-dopa control group $(p>0.05)$. There was no difference between the PD subgroups $(p>0.05)$.

\section{Discussion}

In this study, the recollection of episodic details and the assessment of familiarity during recognition was examined in idiopathic, nondementing, moderate PD patients $\mathrm{ON}$ and OFF their routine dopaminergic medication. Patients were subdivided into two groups according to medication regimen: the index PD subgroup on a dopamine D2 agonist (pramipexole or ropinirole) plus l-dopa (with/without an enzyme inhibito) and a second PD control subgroup on l-dopa (with/without an enzyme inhibitor but no D2 dopamine agonists). Patient performance was compared to a set of demographically and neuropsychologically matched healthy control group.

The key findings can be summarised as follows: Collapsing the PD subgroups (dopamine agonist subgroup and l-dopa control subgroup) into a single group, and combining performance from both medication conditions (ON/Green and OFF/Blue-medication), revealed a selective deficit in recollection with sparing of overall recognition memory and familiarity compared to the healthy controls. When the PD patients were separated into subgroups according to medication regimen, patients in both 

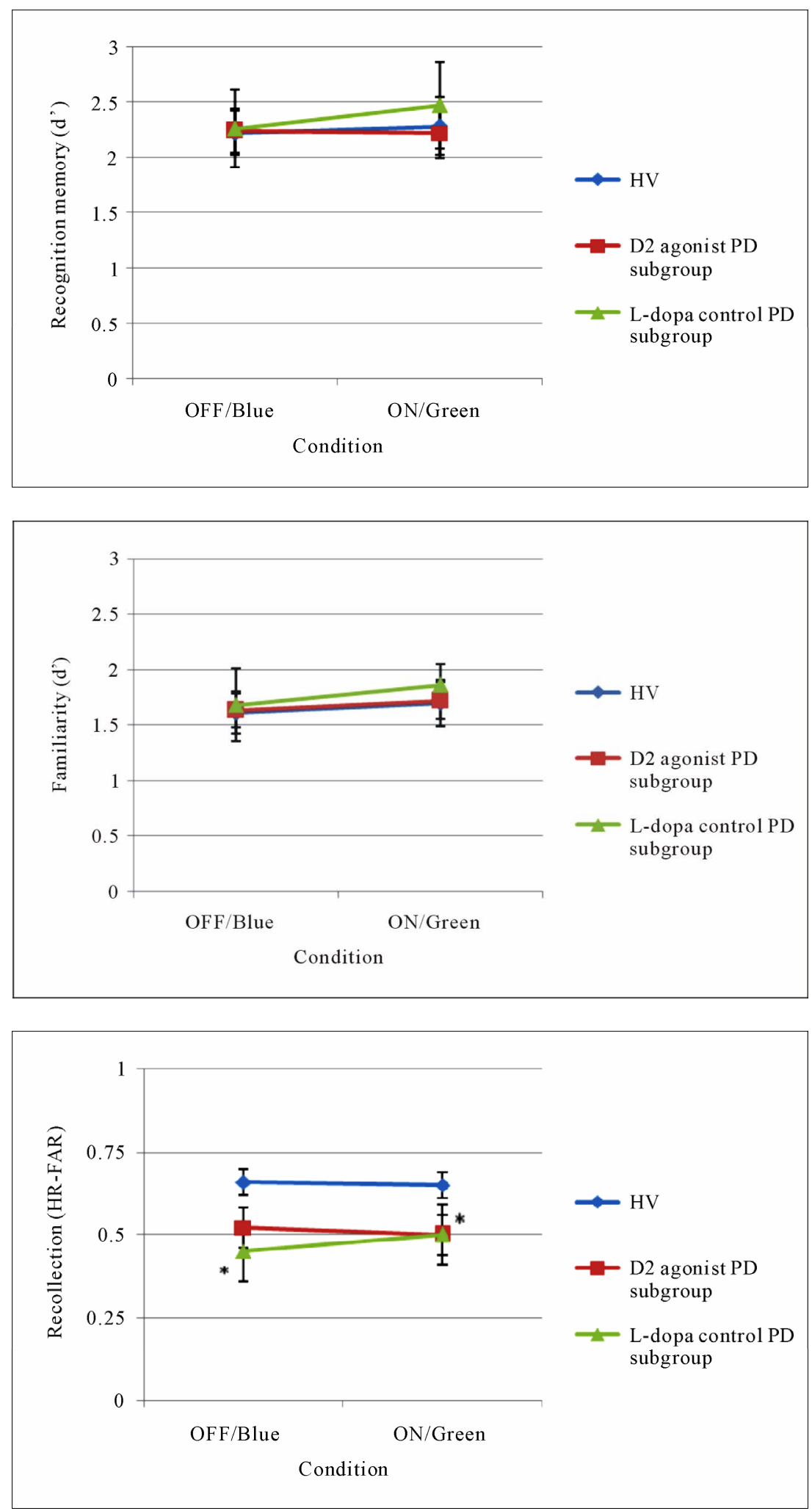

Figure 1. Estimates of ON-medication/Green and OFF-medication/Blue recognition memory (upper), familiarity (middle) and recollection (lower) by participant group. Notes: Error bars represent standard errors of the mean; Abbreviations: $\mathrm{OFF}=$ unmedicated state; $\mathrm{ON}=$ medicated state. Significant at ${ }^{*} p<0.05$. 
the dopamine agonist (plus l-dopa) subgroup and l-dopa (only) control group exhibited significant impairments in recollection. However, when examined in an optimally medicated state the memory profile deviated, with recollection deficits present in only the agonist subgroup. Taken together these findings suggest that the reported PD patients, irrespective of their medication regimen, show impairments in recollection-evident in the unmedicated condition. The severity of this impairment increases when patients are medicated with dopamine agonist (plus l-dopa) but not l-dopa alone. It is unlikely that the differences in the ON-medication profile between the two PD subgroups can be explained by heterogeneity in PD phenotype [69,70], as both patient subgroups were matched for demographic, clinical and IQcharacteristics.

The impact of dopaminergic medication on memory has largely been overlooked in recent years, and this is certainly the case in relation to studies of recollection and familiarity. To date, with the exception of one study [1], recognition memory processes have examined PD patients in a medicated state. This is an important consideration, given the large body of evidence showing that l-dopa, and to a lesser extent pramipexole, can enhance and impair a range of executive functions including working memory and various forms of probabilistic reversal learning. There is good reason to suspect that dopaminergic medication may also affect episodic memory in PD, given the critical role of dopamine in modulating medical temporal lobe memory structures, as well as prefrontal areas which may support strategic memory processes as well.

This study replicates the array of previous studies by reporting a dissociation between relatively spared familiarity and deficient recollection [1,2,4]; the presence of medication-dependent effects on recollection but not familiarity [1]; and, for the first time, that the presence of a disease dependent decline in recollection is increased by dopamine $\mathrm{D} 2$ agonists but not l-dopa.

It could be argued that the negative impact of D2 agonists on recollection is secondary to the sometime reported side-effects of somnolence and reduced vigilance of pramipexole and ropinirole [71,72]. However, this parsimonious explanation fails to stand up to scrutiny, since previous work has shown that D2 agonists, and pramipexole in particular, enhances rather than impairs working memory [53,58,73], and probabilistic reversal learning [53]. Since both working memory and probabilistic reversal learning are cognitively-demanding activities, if pramipexole-induced somnolence were to be a significant factor-since a decline in vigilance would certainly be evident on these tasks.
A related point is the relationship between working memory and recollection. Previous studies have suggested that an impairment in working memory underpins the decline in episodic memory in PD [74,75]. It should follow, therefore, that drugs which enhance working memory [53,58,71] should also exert (secondary) benefits on recollection. Accordingly, if pramipexole improves working memory in PD, and recollection deficits in PD are contingent, at least in part, on working memory deficits, then our D2 agonist group should show improved recollection ON compared to OFF medicationwhich is in fact the opposite to that observed. The implication of these findings are that recollection deficits in PD patients medicated with D2 agonists are not just secondary to a decline in vigilance or working memory, but rather reflect primary memory deficits underpinned by abnormalities at D2 receptor sites in the medial temporal lobe.

In support of this proposal, there is a large body of evidence documenting the presence of D2 receptors in the hippocampus [75-78] and their involvement in memory from studies examining post-mortem tissue [27], brain imaging [76] and injecting quinpirole, a D2 receptor antagonist, into the hippocampus of rats [33].

The smaller group of patients on l-dopa (montherapy or with an adjuvant enzyme inhibitors) showed a different profile across the medication conditions. Rather than falling in line with the D2 agonists, l-dopa appeared to enhance recollection rather than deplete it further. This conclusion is based on the observation that in the OFF state, l-dopa patients' recollection rate was impaired whereas in the ON state, this deficit was remediated and recollection rates in l-dopa and healthy control groups were comparable. This finding supports the predictions arising from the l-dopa overdose hypothesis: that cognitive deficits contingent on dopamine depletion are remediated by l-dopa. Whilst this effect has been reported previously for certain executive functions, this is the first study to report a beneficial effect of l-dopa on recollection memory in PD.

The trend for patients in the l-dopa group to show remediation of their OFF-medication recollection scores when tested in an optimally medicated state (within 3 hours of taking their first l-dopa morning dose) is consistent with other recent studies reporting l-dopa enhances the acquisition and consolidation of an artificial vocabulary in a dose dependent relationship in young healthy males [39], verbal memory in PD [79] and object recognition memory in PD [80]. This effect appears to be limited to declarative memory, with the recent report by Shohamy, Myers, Geghman, Sage and Gluck [49] showing l-dopa disrupts incidental learning, but showed spared 
ability to generalise based on learned information. Cools et al. [47] noted that l-dopa medication remediates cognitive inflexibility but increases impulsivity in Parkinson's. Impulsivity in the current context could be interpreted as showing an increased tendency towards making false alarms. However, inspection of false alarm rates across the three groups in the medicated condition, as well as medicated and unmedicated false alarm rates within the l-dopa group specifically were unaffected by medication type or state.

In conclusion, the results of the current study demonstrate that nondementing moderate Parkinson's can selectively impair the recollection of episodic details during recognition, and provides preliminary evidence that the severity of the recollection deficit is increased by D2 dopamine agonists.

\section{Acknowledgements}

We thank the patients who agreed to delay their medication and their carers who had to support the patients at this time, and the healthy volunteers.

\section{Declaration of Interest}

None of the authors have any actual or potential conflict of interest including any financial, personal or other relationships with other people or organizations within three years of beginning the submitted work that could inappropriately influence, or be perceived to influence, this work.

\section{REFERENCES}

[1] N. M. J. Edelstyn, T. S. Shepherd, A. R. Mayes, S. M. Sherman and S. J. Ellis, "Effect of Disease Severity and Dopaminergic Medication on Recollection and Familiarity in Patients with Idiopathic Nondementing Parkinson's," Neuropsychologia, Vol. 48, No. 5, 2010, pp. 1367-1375. doi:10.1016/j.neuropsychologia.2009.12.039

[2] J. F. Hay, M. Moscovitch and B. Levine, "Dissociating Habit and Recollection: Evidence from Parkinson's Disease, Amnesia and Focal Lesion Patients," Neuropsychologia, Vol. 40, No. 8, 2002, pp. 1324-1334. doi:10.1016/S0028-3932(01)00214-7

[3] N. M. J. Edelstyn, A. R. Mayes, L. Condon, M. Tunnicliffe and S. J. Ellis, "Recognition, Recollection, Familiarity and Executive Function in Patients with Moderate Parkinson's Disease,” Journal of Neuropsychology, Vol. 1, No. 2, 2007, pp. 131-147. doi:10.1348/174866407X182565

[4] J. Barnes, L. Boubert, J. Harris, A. J. Lees and A. S. David, "Reality Monitoring and Visual Hallucinations in Parkinson's Disease,” Neuropsychologia, Vol. 41, No. 5, 2003, pp. 565-574. doi:10.1016/S0028-3932(02)00182-3

[5] K. R. McDonald, A. R. Crossman, A. R. Mayes, J. P. R.
Dick, M. W. Kellett and E. Poliakoff, "Recollection and Familiarity in Parkinson's Disease,” Personal Communication.

[6] P. S. R. Davidson, D. Anaki, J. A. Saint-Cyr, T. W. Chow and M. Moscovitch, "Exploring the Recognition Memory Deficit in Parkinson's Disease: Estimates of Recollection versus Familiarity,” Brain, Vol. 129, No. 7, 2006, pp. 1768-1779. doi:10.1093/brain/awl115

[7] B. Weiermann, M. A. Stephan, A. Kaelin-Lang and B. Meier, "Is There a Recognition Memory Deficit in Parkinson's Disease: Evidence from Estimates of Recollection and Familiarity,” International Journal of Neuroscience, Vol. 120, No. 3, 2010, pp. 211-216. doi:10.3109/00207450903506510

[8] M. Cohn, M. Moscovitch and P. S. R. Davidson, "Double Dissociation between Familiarity and Recollection in Parkinson's Disease as a Function of Encoding Tasks," Neuropsychologia, Vol. 48, No. 14, 2010, pp. 4142-4147. doi:10.1016/j.neuropsychologia.2010.10.013

[9] A. P. Yonelinas, "The Nature of Recollection and Familiarity: A Review of 30 Years of Research," Journal of Memory and Language, Vol. 46, No. 3, 2002, pp. 441-517. doi:10.1006/jmla.2002.2864

[10] J. P. Aggleton and M. W. Brown, "Episodic Memory, Amnesia, and the Hippocampal-Anterior Thalamic Axis," Behavioural Brain Sciences, Vol. 122, 1999, pp. 425-489. doi:10.1017/S0140525X99002034

[11] J. P. Aggleton, J. R. Dumont and E. C. Warburton, “Unraveling the Contributions of the Diencephalon to Recognition Memory: A Review,” Learning and Memory, Vol. 18, 2011, pp. 384-400. doi:10.1101/lm.2167511

[12] N. A. Otmakhova and J. E. Lisman, “ $D_{1} / D_{5}$ Dopamine Receptor Activation Increases the Magnitude of Early Long-Term Potentiation at CA1 Hippocampal Synapses," Journal of Neuroscience, Vol. 16, No. 23, 1996, pp. 7478-7486.

[13] B. G. Mockett, W. M. Brooks, W. P. Tate and W. C. Abraham, "Dopamine $\mathrm{D}_{1} / \mathrm{D}_{5}$ Receptor Activation Fails to Initiate an Activity-Independent Late-Phase LTP in Rat Hippocampus,” Brain Research, Vol. 1021, No. 1, 2004, pp. 92-100. doi:10.1016/j.brainres.2004.06.039

[14] M. A. Wood, M. P. Kaplan, A. Park, E. J. Oliveira, T. L. Lombardi and T. Abel, "Transgenic Mice Expressing a Truncated form of CREB-Binding Protein (CBP) Exhibit Deficits in Hippocampal Synaptic Plasticity and Memory Storage,” Learning and Memory, Vol. 13, 2006, pp. 609617. doi:10.1101/lm.86605

[15] N. Lemon and D. Manahan-Vaughan, "Dopamine $D_{1} / D_{5}$ Receptors Gate the Acquisition of Novel Information through Hippocampus Long-Term Potentiation and Long-Term Depression,” Journal of Neuroscience, Vol. 26, No. 29, 2006, pp. 7723-7729. doi:10.1523/JNEUROSCI.1454-06.2006

[16] D. M. Cummings, A. J. Milnerwood, G. M. Dallérac, V. Waights, J. Y. Brown, S. C. Vatsavayai, C. Hirst and K. P. S. J. Murphy, “Abberant Cortical Synaptic Plasticity and 
Dopaminergic Dysfunction in a Mouse Model of Huntinsdon's Disease," Human Molecular Genetics, Vol. 15, No. 19, 2006, pp. 2856-2868. doi:10.1093/hmg/ddl224

[17] S. W. S. MacDonald, S. Cervenka, L. Farde, L. Nyberg and L. Bäckman, "Extrastriatal Dopamine $\mathrm{D}_{2}$ Receptor Binding Modulates Intraindividual Variability in Episodic Recognition and Executive Functioning," Neuropsychologia, Vol. 47, No. 11, 2009, pp. 2299-2304. doi:10.1016/j.neuropsychologia.2009.01.016

[18] S. Li, W. K. Cullen, R. Anwyl and M. J. Rowan, "Dopamine-Dependent Facilitation of LTP Induction in Hippocampal CA1 by Exposure to Spatial Novelty," Nature Neuroscience, Vol. 6, No. 5, 2003, pp. 526-531. doi:10.1038/nn1049

[19] M. Bentivoglio and M. Morelli, "Dopamine Circuits and Receptors,” In: S. B. Dunnett, M. Bentivoglio, A. Björklund and T. Hökfelt, Eds., Handbook of Chemical Neuroanatomy, Vol. 21, Dopamine, Chapter 1, Elsevier, 2005.

[20] C. M. O’Carroll, S. J. Martin, J. Sandin, B. Frenguelli and R. G. M. Morris, "Dopaminergic Modulation of the Persistence of One-Trial Hippocampus-Dependent Memory,” Learning and Memory, Vol. 13, No. 6, 2006, pp. 760-769. doi:10.1101/lm.321006

[21] B. G. Mockett, D. Guévremont, J. M. Williams and W. C. Abraham, "Dopamine $\mathrm{D}_{1} / \mathrm{D}_{5}$ Receptor Activation Reverses NMDA Receptor-Dependent Long-Term Depression in Rat Hippocampus,” Journal of Neuroscience, Vol. 27, No. 11, 2007, pp. 2918-2926. doi:10.1523/JNEUROSCI.0116-07.2007

[22] U. Frey, H. Schroeder and H. Matthies, "Dopaminergic Antagonists Prevent Long-Term Maintenance of Posttetanic LTP in the CA1 Region of Rat Hippocampal Slices,” Brain Research, Vol. 522, No. 1, 1990, pp. 69-75. doi:10.1016/0006-8993(90)91578-5

[23] U. Frey, H. Matthies and K. G. Reymann, "The Effect of Dopaminergic D1 Receptor Blockade during Tetanization on the Expression of Long-Term Potentiation in the Rat CA1 Region in Vitro,” Neuroscience Letters, Vol. 129, No. 1, 1991, pp. 111-114. doi:10.1016/0304-3940(92)90364-D

[24] R. Bernabeu, L. Bevilaqua, P. Ardenghi, E. Bromberg, P. Schmitz, M. Bianchin, I. Izquierdo and J. H. Medina, "Involvement of Hippocampal cAMP/cAMP-Dependent Protein Kinase Signaling Pathways in a Late Memory Consolidation Phase of aversively Motivated Learning in Rats," Proceedings of the National Academy of Science (USA), Vol. 94, 1997, pp. 7041-7046. doi:10.1073/pnas.94.13.7041

[25] W. Schultz, "Predictive Reward Signal of Dopamine Neurons,” Journal of Neurophysiology, Vol. 80, No. 1, 1998, pp. 1-27.

[26] J. E. Lisman and A. A. Grace, “The Hippocampal-VTA Loop: Controlling the Entry of Information into LongTerm Memory," Neuron, Vol. 46, No. 5, 2005, pp. 703713. doi:10.1016/j.neuron.2005.05.002
[27] N. Kemppainen, M. Laine, M. P. Laakso, V. Kaasinen, V. Någren, T. Vahlber, T. Kurki and J. O. Rinne, "Hippocampal Dopamine $\mathrm{D}_{2}$ Receptors Correlate with Memory Functions in Alheimer's Disease," European Journal of Neuroscience, Vol. 19, 2003, pp. 149-154. doi:10.1046/j.1460-9568.2003.02716.x

[28] A. A. Grace, "Phasic versus Tonic Dopamine Release and the Modulation of Dopamine System Responsivity: A Hypothesis for the Eitiology of Schizophrenia,” Neuroscience, Vol. 41, 1991, pp. 1-24. doi:10.1016/0306-4522(91)90196-U

[29] A. A. Grace, S. B. Floresco, Y. Goto and D. J. Lodge, "Regulation of Firing of Dopaminergicneurons and Control of Goal-Directed Behaviors,” Trends in Neuroscience, Vol. 30, No. 5, 2007, pp. 220-227. doi:10.1016/j.tins.2007.03.003

[30] J. C. Horvitz, "Mesolimbocortical and Nigrostriatal Dopamine Responses to Salient Non-Reward Events," Neuroscience, Vol. 96, No. 4, 2000, pp. 651-656. doi:10.1016/S0306-4522(00)00019-1

[31] W. Schultz, P. Dayan and R. R. Montague, "A Neural Substrate of Prediction and Reward,” Science, Vol. 275, No. 5306, 1997, pp. 1593-1599. doi:10.1126/science.275.5306.1593

[32] W. Schultz, "Getting Formal with Dopamine and Reward,” Neuron, Vol. 36, No. 2, 2002, pp. 241-263. doi:10.1016/S0896-6273(02)00967-4

[33] H. Umegaki, J. Munoz, R. C. Meyer, E. L. Spangler, J. Yoshimura, H. Ikari, A. Iguchi and D. K. Ingram, "Involvement of Dopamine $\mathrm{D}_{2}$ Receptors in Complex Maze Learning and Acetylcholine Release in Ventral Hippocampus of Rats," Neuroscience, Vol. 103, No. 1, 2001, pp. 27-33. doi:10.1016/S0306-4522(00)00542-X

[34] I. Bethus, D. Tse and R. G. M. Morris, "Dopamine and Memory: Modulation of the Persistence of Memory for Novel Hippocampal NMDA Receptor-Dependent Paired Associated,” Journal of Neuroscience, Vol. 30, No. 5, 2010, pp. $1610-1618$. doi:10.1523/JNEUROSCI.2721-09.2010

[35] M. G. Packard and N. M. White, "Memory Facilitation Produced by Dopamine Agonists: Role of Receptor Subtype and Mnemonic Requirements,” Pharmacology, Biochemistry and Behaviour, Vol. 33, 1989, pp. 511-518. doi:10.1016/0091-3057(89)90378-X

[36] A. Marighetto, S. Valerio, N. Philippin, V. BertainaAnglade, C. Drieu La Rochelle, R. Jaffard and P. Morain, "Comparative Effects of the Dopaminergic Agonists Piribedil and Bromocriptine in Three Difference Paradigms in Rodents,” Journal of Pharmacology, Vol. 22, 2008, pp. 511-521. doi:10.1177/0269881107083836

[37] A. I. Hersi, W. Rowe, P. Gaudreau and R. Quirion, “Dopamine $\mathrm{D}_{1}$ Receptor Ligands Modulate Cognitive Performance and Hippocampal Acetylcholine Release in Memory-Impaired Aged Rats,” Neuroscience, Vol. 69, 1996, pp. 1067-1074. doi:10.1016/0306-4522(95)00319-E

[38] A. Flöel, C. Breitenstein, F. Hummel, P. Celnik, C. Gin- 
gert, L. Sawaki, S. Knecht and L. G. Cohen, "Dopaminergic Influences on Formation on a Motor Memory," Annals of Neurology, Vol. 58, No. 1, 2005, pp. 121-130. doi:10.1002/ana.20536

[39] S. Knecht, C. Breitenstein, S. Bushuven, S. Wailke, S. Kamping, A. Flöel, A. Zwitselood and E. Berd Ringelstein, "Levodopa: Faster and Better Word Learning in Normal Humans,” Annals of Neurology, Vol. 56, 2005, pp. 20-26. doi:10.1002/ana.20125

[40] A. Flöel, G. Garraux, B. Xu, C. Breitenstein, S. Knecht, P. Herscovitch and L. G. Cohen, "Levodopa Increases Memory Encoding and Dopamine Release in the Striatum in the Elderly," Neurobiology of Aging, Vol. 29, No. 2, 2008, pp. 267-279.

doi:10.1016/j.neurobiolaging.2006.10.009

[41] K. W. Lange, T. W. Robbins, C. D. Marsden, M. James, A. M. Owen and G. M Paul, "L-Dopa Withdrawal in Parkinson's Disease Selectively Impairs Cognitive Performance in Tests Sensitive to Frontal Lobe Dysfunction," Psychopharmacology, Vol. 107, No. 2-3, 1992, pp. 394404. doi:10.1007/BF02245167

[42] S. J. G. Lewis, R. Cools, T. W. Robbins, A. Dove, R. A. Barker and A. M. Owen, "Using Executive Heterogeneity to Explore the Nature of Working Memory Deficits in Parkinson's Disease," Neuropsychologia, Vol. 41, No. 6, 2003, pp. 645-654. doi:10.1016/S0028-3932(02)00257-9

[43] A. Costa, A. Peppe, G. Dell’Agnello, G. A. Carlesimo, L. Murri and U. Bonuccelli, "Dopaminergic Modulation of Visuospatial Working Memory in Parkinson's Disease," Dementia and Geriatric Cognitive Disorders, Vol. 15, No. 2, 2003, pp. 55-66. doi:10.1159/000067968

[44] N. Fournet, O. Moreaud, J. L. Roulin, B. Naegele and J. Pellat, "Working Memory Functioning in Medicated Parkinson's Disease Patients and the Effect of Withdrawal of Dopaminergic Medication,” Neuropsychology, Vol. 14, No. 2, 2000, pp. 247-253. doi:10.1037/0894-4105.14.2.247

[45] C. Malapani, B. Rakitin, W. H. Meck, B. Deweer, B. Dubois and J. Gibbon, "Coupled Temporal Memories in Parkinson's Disease: A Dopamine-Related Dysfunction,” Journal of Cognitive Neuroscience, Vol. 10, 1998, pp. 316-331. doi:10.1162/089892998562762

[46] R. Cools, R. A. Barker, B. J. Sahakian and T. W. Robbins, “Enhanced or Impaired Cognitive Function in Parkinson's Disease as a Function of Dopaminergic Medication and Task Demands," Cerebral Cortex, Vol. 11, No. 2, 2001, pp. 1136-1143. doi:10.1093/cercor/11.12.1136

[47] R. Cools, R. A. Barker, B. J. Sahakian and T. W. Robbins, "L-Dopa Medication Remediates Cognitive Flexibility, but Increases Impulsivity in Patients with Parkinson's Disease," Neuropsychologia, Vol. 41, No. 11, 2003, pp. 1431-1441. doi:10.1093/cercor/11.12.1136

[48] A. E. Hayes, M. C. Davidson and S. W. Keele, "Towards a Functional Analysis of the Basal Ganglia," Journal of Cognitive Neuroscience, Vol. 1, 1998, pp. 178-198. doi:10.1162/089892998562645
[49] D. Shohamy, C. E. Myers, K. D. Geghman, J. Sage and M. A. Gluck, "L-Dopa Impairs Learning, But Spared Generalisation, in Parkinson's Disease,” Neuropsychologia, Vol. 44, No. 5, 2006, pp. 774-784. doi:10.1016/j.neuropsychologia.2005.07.013

[50] A. M. Gotham, R. G. Brown and C. D. Marsden, "Frontal Cognitive Function in Patients with Parkinson's Disease 'ON' and 'OFF' Levodopa,” Brain, Vol. 111, No. 2, 1988, pp. 299-321. doi:10.1093/brain/111.2.299

[51] V. Czernecki, B. Pillon, J. L. Houeto, J. B. Pochon, R. Levy and B. Dubois, "Motivation, Reward and Parkinson's Disease: Influence of Dopatherapy," Neuropsychologia, Vol. 40, No. 13, 2002, pp. 2257-2267. doi:10.1016/S0028-3932(02)00108-2

[52] J. Kulisevsky, A. Avila, M. Barbano, R. Antonijoan, M. Berthier and A. Gironell, "Acute Effects of Levodopa on Neuropsychological Performance in Stable and Fluctuating Parkinson's Disease Patients at Different Levodopa Plasma Levels,” Brain, Vol. 119, 1996, pp. 2121-2132. doi:10.1093/brain/119.6.2121

[53] R. Cools, L. Altamirano and M. D’Esposito, "Reversal Learning in Parkinson's Disease Depends on Medication Status and Outcome Valence,” Neuropsychologia, Vol. 44, No. 10, 2006, pp. 1663-1673. doi:10.1016/j.neuropsychologia.2006.03.030

[54] J. B. Rowe, L. Hughes, B. C. P. Ghosh, D. Eckstein, C. H. Williams-Gray, S. Fallon, R. A. Barker and A. M. Owen, "Parkinson's Disease and dopaminergic Therapy: Differential Effects on Movement, Reward and Cognition," Brain, Vol. 131, No. 8, 2008, pp. 2094-2105. doi:10.1093/brain/awn112

[55] J. A. Cooper, H. J. Sagar, S. M. Doherty, N. Jordan, P. Tidswell and E. V. Sullivan, "Different Effects of Dopaminergic and Anticholinergics Therapies on Cognitive and Motor Function in Parkinson's Disease,” Brain, Vol. 115, No. 6, 1992, pp. 1701-1725. doi:10.1093/brain/115.6.1701

[56] E. Driver-Dunckley, J. Samanta and M. Stacy, "Pathological Gambling Associated with Dopamine Agonist Therapy in Parkinson's Disease,” Neurology, Vol. 61, No. 3, 2003, pp. 422-423. doi:10.1212/01.WNL.0000076478.45005.EC

[57] D. L. Dodd, K. J. Klos, J. H. Bower, Y. E. Geda, K. A. Josephs and J. E. Ahlskog, "Pathological Gambling Caused by Drugs Used to Treat Parkinson's Disease,” Archives of Neurology, Vol. 62, No. 9, 2005, pp. 13771381. doi:10.1001/archneur.62.9.noc50009

[58] A. Costa, A. Peppe, G. Dell’Agnello, C. Caltagirone and G. A. Carlesimo, "Dopamine and Cognitive Functioning in De Novo Subjects with Parkinson's Disease: Effects of Pramipexole and Pergolide on Working Memory," Neuropsychologia, Vol. 47, No. 5, 2009, pp. 1374-1381. doi:10.1016/j.neuropsychologia.2009.01.039

[59] M. M. Hoehn and M. D. Yahr, "Parkinsonism: Onset, Progression and Mortality,” Neurology, Vol. 17, No. 5, 1967, pp. 427-442. 
[60] H. E. Nelson and J. Willison, "National Adult Reading Test,” Windsor, Berks, NFER-Nelson, 1991.

[61] M. F. Folstein, S. E. Folstein and P. R. McHugh, "Mini-Mental State: A Practical Method for Grading the Cognitive State of Patients for the Clinician,” Journal of Psychiatric Research, Vol. 12, No. 3, 1975, pp. 189-198. doi:10.1016/0022-3956(75)90026-6

[62] M. Roth, F. A. Huppert, C. Q. Mountjoy and E. Tym, "CAMDEX-R: The Cambridge Examination for Mental Disorders of the Elderly-Revised," Cambridge University Press, Cambridge, 1998.

[63] W. M. Reynolds and K. A. Kobak, "Hamilton Depression Inventory: A Self-Report Version of the Hamilton Depression Rating Scale,” Psychological Assessment Resources, Odessa, 1995.

[64] S. Fahn and R. L. Elton, “The Unified Parkinson's Disease Rating Scale,” In: S. Fahn, C. D. Marsden, D. B. Calne and M. Goldstein, Eds., Recent Developments in Parkinson's Disease, Macmillan, Florham Park, 1987, pp. 293-304.

[65] M. Coltheart, "The MRC Psycholinguistic Database," Quarterly Journal of Experimental Psychology, Vol. 33A, 1981, pp. 497-505.

[66] R. H. Baayen, R. Piepenbrock and H. van Rijn, "The CELEX Lexical Database (CD-ROM)," Linguistic Data Consortium, University of Pennsylvania, Philadelphia, 1993.

[67] J. G. Snodgrass and J. Corwin, "Pragmatics of Measuring Recognition Memory. Applications to Dementia and Amnesia," Quarterly Journal of Experimental Psychology, Vol. 117, No. 1, 1988, pp. 34-50. doi:10.1037/0096-3445.117.1.34

[68] A. P. Yonelinas and L. L. Jacoby, “The Relation between Remembering and Knowing as Bases for Recognition: Effects of Size Congruency," Journal of Memory and Language, Vol. 34, No. 5, 1995, pp. 622-643. doi:10.1006/jmla.1995.1028

[69] S. J. Lewis, T. Foltynie, A. D. Blackwell, T. W. Robbins, A. M. Owen and R. A. Barker, "Heterogeneity of Parkinson's Disease in the Early Clinical Stages Using a Data Driven Approach,” Journal of Neurology, Neurosurgery and Psychiatry, Vol. 76, No. 3, 2005, pp. 343-348. doi:10.1136/jnnp.2003.033530

[70] M. Selikova, D. R. Williams, P. A. Kempster, J. L. Holton, T. Revesz and A. J. Lees, "A Clinic-Pathological Study of Subtypes in Parkinson's Disease,” Brain, Vol. 132, No. 11, 2009, pp. 2947-2957. doi:10.1093/brain/awp234

[71] R. A. Hauser, L. Gauger, W. M. Anderson and T. A. Zesiewicz, "Pramipexole-Induced Somnolence and Epi- sodes of Daytime Sleep,” Movement Disorders, Vol. 15, No. 4, 2000, pp. 658-663. doi:10.1002/1531-8257(200007)

[72] W. Ondo, R. Fayle, F. Atassi and J. Jankovic, "Modafinil for Daytime Somnolence in Parkinson's Disease: Double Blind, Placebo Controlled Parallel Trial,” Journal of Neurology, Neurosurgery and Psychiatry, Vol. 76, No. 12, 2005, pp. 1636-1639. doi:10.1136/jnnp.2005.065870

[73] D. M. Barch, "Pharmacological Manipulation of Human Working Memory,” Psychopharmacology (Berl), Vol. 174, No. 1, 2004, pp. 126-135. doi:10.1007/s00213-003-1732-3

[74] J. D. E. Gabrieli, J. Singh, G. T. Stebbins and C. G. Goetz, "Reduced Working Memory Span in Parkinson's Disease: Evidence for the Role of a Frontostriatal System in Working and Strategic Memory,” Neuropsychology, Vol. 10, No. 3, 1996, pp. 322-332. doi:10.1037/0894-4105.10.3.321

[75] J. H. Meador-Woodruff, S. P. Damask and S. J. Watson Jr. "Differential Expression of Autoreceptors in the Ascending Dopamine Systems of the Human Brain,” Proceedings of the National Academy Science (USA), Vol. 91, 1994, pp. 8297-8301. doi:10.1073/pnas.91.17.8297

[76] M. Matsumoto, K. Hidaka, S. Tada, Y. Tasaki and T. Yamaguchi, "Full-Length cDNA Cloning and Distribution of Human Dopamine $\mathrm{D}_{4}$ Receptor," Molecular Brain Research, Vol. 29, No. 1, 1995, pp. 157-162. doi:10.1016/0169-328X(94)00245-A

[77] L. Mrzljak, C. Bergson, M. Pappy, R. Huff, R. Levenson and P. S. Goldman-Rakic, "Localization of Dopamine $\mathrm{D}_{4}$ Receptors in GABAergic Neurons of the Primate Brain,” Nature (Lond), Vol. 381, 1996, pp. 245-248. doi:10.1038/381245a0

[78] H. Takahashi, M. Kato, M. Hayashi, Y. Okubo, A. Takano, H. Ito and T. Suhara, "Memory and Frontal Lobe Functions: Possible Relations with Dopamine $\mathrm{D}_{2}$ Receptors in the Hippocampus," NeuroImage, Vol. 34, No. 4, 2007, pp. 1643-1649. doi:10.1016/j.neuroimage.2006.11.008

[79] E. Mohr, G. Fabbrini, J. Williams, J. Schlegel, C. Cox, P. Fedio and T. N. Chase, "Dopamine and Memory Function in Parkinson's Disease,” Movement Disorders, Vol. 4, 2004, pp. 113-120. doi:10.1002/mds.870040202

[80] S. Righi, M. P. Viggiano, M. Paganini, S. Ramat and P. Marini, "Recognition of Category-Related Visual Stimuli in Parkinson's Disease: Before and after Pharmacological Treatment," Neuropsychologia, Vol. 45, No. 13, 2007, 2931-2941. doi:10.1016/j.neuropsychologia.2007.06.002 